\title{
Experience from two decades of the Cambridge Rapid Access Neurology Clinic
}

\author{
Authors: Laura T Axinte, ${ }^{\mathrm{A}}$ Barnaby D Fiddes, ${ }^{\mathrm{B}}$ Alastair Donaghy, ${ }^{\mathrm{C}}$ Adam Whyte, ${ }^{\mathrm{D}}$ Chris Allen, ${ }^{\mathrm{E}}$ Stephen J Sawcer, ${ }^{\mathrm{F}}$ \\ Robert J Adam ${ }^{G}$ and Sybil RL Stacpoole ${ }^{H}$
}

\begin{abstract}
We report on the evolution of the rapid access neurology clinic (established in 1995) at Addenbrooke's Hospital, Cambridge. Annualised attendance data demonstrate an ever increasing demand, with primary headache disorders now accounting for more than $40 \%$ of referrals. Secondary causes of headache (including intracranial tumours, idiopathic intracranial hypertension, carotid or vertebral artery dissection and subdural haematomas) remain infrequent. In all such cases, there were additional diagnostic clues. The number of patients referred with problems related to chronic neurological diseases has fallen considerably, reflecting the roles of specialist nurses and clinics. Imaging investigation of choice shifted from computerised tomography scan ( 45 to $16 \%$ ) towards magnetic resonance imaging (17 to $47 \%$ ). Management is increasingly on an outpatient basis, often without the need for a follow-up appointment. The experience presented here should inform further development of rapid access neurology clinics across the UK and suggests the need for acute headache services, in line with those for transient ischaemic attack and first seizure.
\end{abstract}

KEYWORDS: Acute neurology, headaches, rapid access

\section{Introduction}

Acute neurological presentations are a common problem, accounting for around $17 \%$ of general practitioner (GP) consultations, $10 \%$ of accident and emergency (A\&E) visits and $20 \%$ of acute medical admissions. ${ }^{1,2}$ There is increasing appreciation of the requirement to reconfigure neurological services to meet this need, but the challenge is to provide a

Authors: AFY1, Barnet General Hospital, London, UK; Bneurology SpR, Addenbrooke's Hospital, Cambridge University Hospitals NHS Foundation Trust, UK; CFY2, Royal Victoria Hospital, Belfast, UK; DSHO, John Radcliffe Hospital, Oxford, UK; E' Consultant neurologist, Addenbrooke's Hospital, Cambridge University Hospitals NHS Foundation Trust, UK; Fprofessor of neurology, Addenbrooke's Hospital, Cambridge University Hospitals NHS Foundation Trust, UK; ${ }^{G}$ Consultant neurologist, Norfolk and Norwich University Hospitals NHS Foundation Trust, Norwich, UK; ${ }^{H}$ consultant neurologist, Addenbrooke's Hospital, Cambridge University Hospitals NHS Foundation Trust, UK service for those who most need it with an under-resourced specialty. ${ }^{3}$ The Royal College of Physicians' (RCP's) 2012 consultant physician survey reported that there were 716 neurology consultants in the UK, resulting in 1 per 90,000 population, significantly lower than the recommended RCP minimum of 1 consultant per 70,000. ${ }^{3,4}$ However, the current figure compares favorably to 1998, when there were only 290 neurologists for a UK population of 60 million (less than 1 per 200,000). There remains considerable geographic variability across the country, with the east of England having 1 consultant per 140,000 people, whereas north-central/north-east London have at least 1 per $50,000 .^{5}$

Due to the current shortage of consultant neurologists and the regional variability in accessing their services, many patients admitted with neurological symptoms, especially in district general hospitals, are likely to be treated by general physicians and not receive specialist neurology assessment. ${ }^{3,6}$ However, timely involvement of a neurologist can lead to a change in diagnosis or management in as many as $79 \%$ of cases. ${ }^{7-9}$ At the same time, the political drive to reduce waiting times for hospital outpatient appointments has contributed to an increased demand for neurology clinic appointments which has not been matched by a proportional increase in the number of trained neurologists. Rapid access clinics have been proposed as a solution to this shortage, allowing patients with neurological symptoms to see a neurologist promptly for an assessment, appropriate investigations and treatment. ${ }^{10-12}$

In response to the growing pressure for urgent neurological assessment from local GPs, a rapid access neurology clinic was introduced at Addenbrooke's Hospital in February 1995. This service remains 20 years later and is now part of a 'rapid access neurology service' which includes a first seizure clinic and a transient ischaemic attack (TIA) service in addition to the more traditional '24/7' availability of a specialist registrar (SpR) for telephone advice and ward assessment of inpatients. Similar rapid access clinics are now widespread across the UK, with a 2014 Association of British Neurologists' survey showing that all tertiary neuroscience centres provide an acute neurology clinic, although figures are dramatically lower outside such centres. ${ }^{3}$ Further expansion of such emergency clinics may be of value since it may lead to the initiation of suitable investigations more promptly, more accurate diagnoses and may even reduce hospital admissions. ${ }^{7-9,13}$

This report brings together three audits of the rapid access neurology clinic at Addenbrooke's Hospital, spanning almost 
two decades. ${ }^{10,14}$ Our data demonstrate evolution in patterns of referral, patient presentation, investigation and management, some of which also inform and/or reflect changes in the neurology service.

\section{Methods}

The neurology unit at Addenbrooke's Hospital in Cambridge (a teaching hospital and tertiary neuroscience centre) provides care for Cambridge and Huntingdon Health Authority. This covers a population of 635,000 (as estimated by Cambridgeshire County Council Research Group for mid-2013), an increase from 450,000 in $1997 .^{15}$

Since its establishment in February 1995, appointments for the rapid access neurology clinic have been made following discussion between the referring clinician and the neurology registrar (SpR) on call. Initially, referrals were accepted from GPs only, but when indicated, referrals from other specialties such as the A\&E department and general medicine are now accepted. Appointments are 30 minutes long, and have increased from 8 per week in 1995 ( 2 per day, between 8 and 9 am, Tuesday to Friday) to 13 per week since autumn 2013 (4 each in regular clinics on Monday, Tuesday and Thursday and 1 on Friday). Patients are telephoned and offered the next available appointment. Clinics are provided by a neurology $\mathrm{SpR}$, with supervision by the neurology consultant on call. There is access to on the day computerised tomography (CT) imaging and magnetic resonance imaging (MRI) within 2 weeks (when requested urgently).

Data were collected retrospectively from patients attending the rapid access neurology clinic during the specified time periods: 1 February 1995 - 1 August 1996 (18 months), ${ }^{10}$ 1 January 2008 - 31 December 2009 (2 years), ${ }^{14}$ and for this the most recent study 1 February 2014 - 1 August 2014 (6 months). (Referrals to first seizure clinic and TIA clinic follow separate pathways and were not considered in these audits.)

For the first two audit periods, a paper diary record was kept of all patients attending the clinic. In 2014, patients were identified from hard-copy clinic templates and electronically cross-referenced by checking all clinic codes active during that period. For the initial audit, the data were compiled from reviewing the paper notes; for the two subsequent audits, the information was located from clinic letters, investigation reports and results letters stored in the electronic medical record, picture archiving and communication system and paper notes.

In order to allow easier comparison across the three audit periods, the results shown in figures and tables have been adjusted to provide annualised figures.

\section{Results}

\section{Increasing demand for rapid access}

During the 6-month period (1 February - 1 August 2014), 214 rapid access clinic appointments were made (428 patient episodes per annum). In total, 13 patients did not attend (DNA) and 7 others were excluded from the analysis due to either cancelling the appointment or not meeting the criteria for clinic attendance (eg follow-up appointment rather than a new presentation), leaving 194 patients included in the analysis
Table 1. Diagnostic classification of patients

presenting to the neurology emergency clinic

(annualised data).

\section{Diagnostic classification}

1995-6 2008-9 2014

Migraine

Headache not migraine

17.0

37.5

102.0

Multiple sclerosis

$34.0 \quad 31.5 \quad 56.0$

Possible multiple sclerosis

$29.0 \quad 16.5$

12.0

Functional

$\begin{array}{lll}13.0 & 7.5 & 4.0\end{array}$

Cerebral tumour

Peripheral neuropathy

$17.0 \quad 11.5$

18.0

Peripheral nerve lesion

$\begin{array}{ll}7.0 & 10.5\end{array}$

4.0

Dizziness

$\begin{array}{lll}2.0 & 9.0 & 0.0\end{array}$

Stroke

$5.0 \quad 7.0$

16.0

Transient ischaemic attack

2.0

8.5

14.0

Epilepsy

$\begin{array}{lll}10.0 & 6.0 \quad 4.0\end{array}$

Faint/collap

(collapse

23.0

6.0

Cervical disc disease

$4.0 \quad 2.0$

Structural myelopathy

$5.0 \quad 4.5$

6.0

Lumbosacral disc disease

$5.0 \quad 2.0$

4.0

Myasthenia

$2.0 \quad 1.0$

8.0

Dementia

$1.0 \quad 2.5$

10.0

Cranial nerve disease

1.0

Idiopathic intracranial hypertension

Motor neuron disease

$1.0 \quad 2.0$

2.0

$\begin{array}{lll}7.0 & 1.5 & 2.0\end{array}$

$\begin{array}{lll}0.0 & 2.5 & 8.0\end{array}$

CNS infection

$\begin{array}{lll}1.0 & 1.0 \quad 8.0\end{array}$

CNS infection

$4.0 \quad 0.5$

4.0

Transient global amnesia

$2.0 \quad 0.0$

4.0

Parkinson's disease

$\begin{array}{lll}1.0 & 0.0 & 2.0\end{array}$

Facial pain

3.0

Other neurological

Other non-neurological

13.0

1.5

2.0

Uncertain

$\begin{array}{lll}0.0 & 11.5 \quad 24.0\end{array}$

Total

14.0

8.0

16.0

CNS = central nervous system.

(or 388 episodes per annum). This is a marked increase (86\%) from the numbers seen in 2008-2009 (415 patients in 2 years or 207.5 episodes per annum) and 1995-1996 (350 patients in 18 months or 233 episodes per annum) (Table 1). At its inception in 1995, it was intended that patients would be seen within 24 hours of referral; however, the increasing pressure on the service means that this target is now rarely met. As a result, the service provided would now be described as rapid access (two-week wait) rather than emergency (next day). Equally, patients sometimes choose an appointment that is not necessarily the next one available, for reasons that include child care availability and work commitments. In 2014, only $16 \%$ of referrals were seen within 48 hours and the average wait to 
be seen was 8 days; $92 \%$ of patients were seen within 2 weeks. In keeping with the increased wait, DNA rates have increased slightly to $6.2 \%$ in 2014 from $2.5 \%$ in $1995-1996$. This is consistent with the average Cambridge University Hospitals NHS Foundation Trust DNA rate for outpatient appointments (5.2\% in 2012-2013), ${ }^{16}$ which is perhaps surprising considering the emergent nature of this clinic.

In 2014, the main source of referral remained general practice (89\%), followed by A\&E (8\%), ophthalmology (1\%) and general medicine $(1 \%)$; all other specialties combined contributed the remaining $1 \%$. Initially, $100 \%$ of the referrals were from general practice, but the flexibility to take referrals from other sources has developed.

Patient demographics did not change significantly over the three audit periods, except that a higher proportion of patients presenting in the most recent audit period were male than previously. In 2014, the mean age of patients attending the clinic was 45.8 years (range 17-88; F:M 1.28). In 2008-09 this was 43.6 years (range 16-94; F:M 1.72) and in 1995-96, the mean age was 44.2 years (range $13-81 ; \mathrm{F}: \mathrm{M} 1.73$ ).

\section{Changing presentations}

Headache was the most common reason for referral in all audit periods, increasing from $27 \%$ in $1995-96$ to $39 \%$ in 2008-09 and to $51 \%$ in 2014. However, as in both previous audits, headache without signs remains invariably benign. In the 2014 six-month audit period, only 2 patients (1\%) were found to have intracerebral tumours; neither of these presented with headache and both had abnormalities on neurological examination (ataxia, weakness or cognitive impairment). In the 2008-9 two-year audit period, 21 patients (5\%) were found to have intracerebral tumours of whom 7 had headaches among their presenting symptoms, but all also had abnormalities on examination (including visual field deficits, papilloedema, weakness, ataxia or cranial nerve palsies). In the 1995-6 18-month audit, 4 out of 95 patients presenting with headaches had a structural lesion and these also had associated neurological signs. Other secondary causes of headache across the three audits (a total of 959 patients of which 388 patients reported headaches) included idiopathic intracranial hypertension (9), vertebral or carotid dissection (4), subdural haematomas (2), temporal arteritis (1) and a parotid mass pressing on the carotid artery causing the patient to present with headache and Horner's syndrome.

A wide spectrum of neurological conditions presented to the clinic (Table 1 and Fig 1). Considering all patients referred between February and August 2014, a neurological cause for symptoms was identified in $85 \%$ (165) of patients. A non-neurological diagnosis was made in 6\% (12) and a further 5\% (9) were thought to have a functional disorder. The diagnosis was uncertain in the remaining 4\% (8) of patients. The most common diagnosis was migraine in 51 (26\%) patients. Non-migrainous headaches were also very common, and were diagnosed in a further $28(14 \%)$ patients (Table 2). 4 (2\%) patients were diagnosed with idiopathic intracranial hypertension; some of these had headaches, but visual disturbance was the main reason for referral. Apart from headaches, other diagnoses included $8(4 \%)$ patients with multiple sclerosis (MS) or possible MS, 5 (3\%) with myasthenia gravis and $6(3 \%)$ with epilepsy.

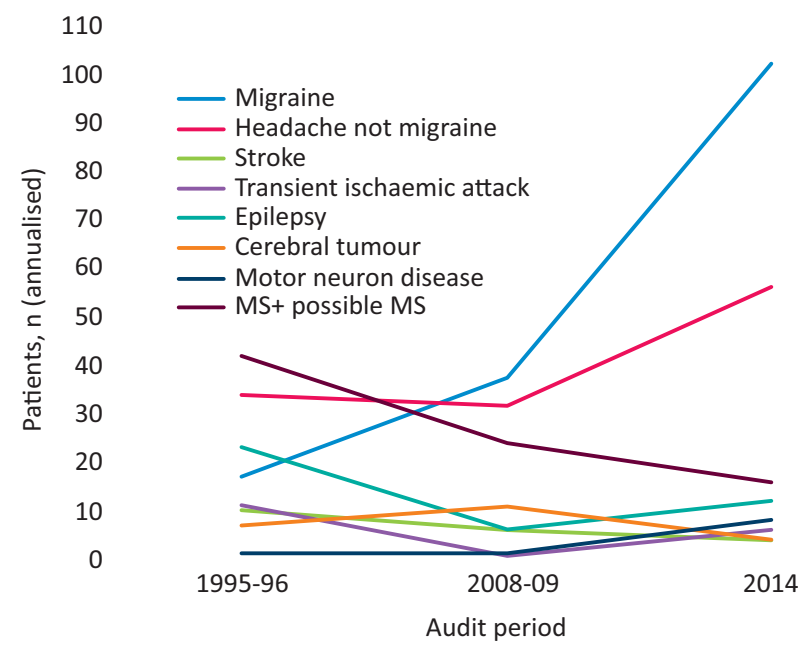

Fig 1. Diagnostic classification (annualised data) trends over time. $\mathrm{MS}=$ multiple sclerosis.

\section{Investigations and management}

In the 2014 audit, 155 (80\%) of patients attending the emergency neurology clinic were investigated. The most common investigation was an MRI (47\%), followed by blood tests (32\%), CT scan (16\%) and electromyography/ nerve conduction studies (14\%) (Table 3). This compares to $45 \%$ having a CT and 17\% an MRI in 1995.

Outcomes for the patients in the 2014 audit were such that almost all were managed as outpatients: only $4(2 \%)$ patients were admitted within 1 week and $1(0.5 \%)$ patient was admitted at a later date for planned investigations. In total, $69(36 \%)$ patients were followed up, $41(21 \%)$ were given treatment and discharged and $82(42 \%)$ were discharged with

\section{Table 2. Classification of primary headache}

disorders presenting to the neurology emergency clinic in 2014.

$\begin{array}{lll}\text { Headache type } & \begin{array}{l}\text { Annualised } \\ \text { number }\end{array} & \begin{array}{l}\text { Total primary } \\ \text { headaches, \% }\end{array} \\ \text { Migraine } & 102 & 64.6 \\ \text { Cluster } & 10 & 6.3 \\ \text { Tension/chronic daily } & 10 & 6.3 \\ \text { Unclassified } & 12 & 7.6 \\ \text { Post-traumatic } & 6 & 3.8 \\ \text { Analgesia overuse } & 4 & 2.5 \\ \text { Cervicogenic } & 4 & 2.5 \\ \text { Neuropathic } & 4 & 2.5 \\ \text { Hemicrania continua } & 2 & 1.3 \\ \text { Sinusitis } & 2 & 1.3 \\ \text { Systemic infection } & 2 & 1.3 \\ \text { Total } & \mathbf{1 5 8} & \end{array}$




\begin{tabular}{|c|c|c|c|}
\hline Investigation & $1995-6$ & $2008-9$ & 2014 \\
\hline CT & 104 & 74 & 64 \\
\hline MRI & 39 & 122 & 182 \\
\hline EEG & 29 & 18 & 12 \\
\hline VEP & 16 & 1 & 4 \\
\hline EMG/NCS & 9 & 15 & 56 \\
\hline CSF & 25 & 12 & 26 \\
\hline MRA & 8 & 9 & 12 \\
\hline Carotid doppler & 6 & 4 & 12 \\
\hline Angiogram & 2 & 0 & 0 \\
\hline Blood tests & 85 & 60 & 126 \\
\hline No investigations & 69 & 105 & 78 \\
\hline \multicolumn{4}{|l|}{ Action taken } \\
\hline Admit within one week & 45 & 15 & 8 \\
\hline Admit as routine & 12 & 3 & 2 \\
\hline Follow up & 138 & 104 & 138 \\
\hline Treatment and discharge & 23 & 29 & 82 \\
\hline Discharge no treatment & 69 & 33 & 164 \\
\hline Refer to specialty & 29 & 20 & 62 \\
\hline
\end{tabular}

no specific treatment or follow up. 31 (16\%) were referred to another specialty (Table 3 ). The most common referral was to neurosurgery (8), followed by ophthalmology (6), psychiatry (3) and ear, nose, and throat (3). The remaining referrals were to other medical (9) and surgical (2) specialties.

By comparison, in 1995-96, 19\% were admitted that day, and $5 \%$ at a later point; $59 \%$ were followed up; $35 \%$ were given treatment and discharged; $31 \%$ were discharged with no specific treatment or follow up and $12 \%$ were referred to another specialty. This change may reflect improved availability of outpatient investigation and/or a change in the type of patients being referred (ie the increase in those presenting with primary headache disorders).

\section{Discussion}

Over the two decades since the rapid access neurology clinic was first set up, the service has expanded significantly to meet demand, increasing capacity by almost two-thirds, as well as developing specialist pathways and clinics to deal with the more common acute neurological presentations. The main source of referral remains general practice, although in recent times a small proportion of referrals have originated from out-of-hours attendances at $\mathrm{A} \& \mathrm{E}$.

Reviewing the diagnostic classification of patients attending the clinic, primary headache disorders predominate $(41 \%$ presentations): since 1995, there has been a $200 \%$ absolute increase in the number of patients with headaches seen in this clinic. The largest increase comes from patients with migraines rather than other non-migrainous headaches (from 17 per year in 1995-96 to 102 per year in 2014). Data from other rapid access clinics across the country have had similar findings: in Belfast, a rapid access clinic piloted over 4 weeks showed that headache was the main complaint for $49 \%$ of patients attending. ${ }^{11}$ In Edinburgh, 25\% of the rapid access clinic attendances were due to headache. ${ }^{12}$

With regard to other diagnoses, there has been a steady decline in the number of patients with MS or possible MS (42 per year in 1995-96 to 16 per year in 2014), epilepsy (23 per year in 1995-96 to 12 per year in 2014) and stroke/TIA (21 per year in 1995-96 to 10 per year in 2014) seen in this clinic. This decrease reflects the availability of MS, epilepsy and stroke specialist nurses, and the establishment of separate first seizure and TIA clinics.

In keeping with the increasing reliance on and availability of MRI, significantly more MRI than CT scans were performed in 2014, the numbers almost mirroring the requests in 199596. Compared to 1995-96, a higher proportion of patients attending this clinic were investigated (66.1 vs $80 \%)$ and it is likely that this also is partly due to the greater availability of MRI, whereas the benefits of ordering a CT scan had to be carefully balanced against its high radiation dose, especially in younger patients. Other contributory factors may include both patient demand and more defensive medical practice.

The design of this rapid access clinic allows access to CT imaging on the day and MRI within 2 weeks. Given the increasing role for and availability of MRI, there may be a case to incorporate on the day access to MRI into the design of rapid access neurology clinics, in the same way that it can be accessed through TIA clinics in appropriate circumstances.

Over the years, management plans emerging from the rapid access clinic have also changed, with far fewer patients being admitted to hospital and many more being seen once and discharged, with or without treatment (Table 3). The reasons behind these changes are not clear but likely reflect the changing patterns of conditions seen (ie more primary headache disorders), the greater ability to carry out investigations as an outpatient or day case, and the NHS drive to manage more conditions as part of an ambulatory pathway. ${ }^{18}$

Our experience in Cambridge of providing a rapid access neurology clinic over the last two decades may be helpful in the current healthcare climate, with its focus on acute neurological services and increased provision of services in primary care. It is notable that the initial provision of an emergency, next day service has become a rapid access, two-week wait clinic, likely reflecting ever increasing demand and pressures elsewhere in the system. The relative burden of primary headache disorders presenting to this clinic, reflected also in Belfast and Edinburgh, perhaps highlights an opportunity for further improvement in these services, focusing on a pathway for the management of acute headache. There is scope for this to be provided in primary or secondary care; GPs with a special interest in headaches are often able to manage headaches with high patient satisfaction and, increasingly, GPs have direct access to specialist imaging. ${ }^{19}$ Such service redesign may better meet the needs of patients, general practitioners and neurologists. 


\section{References}

1 Royal College of Physicians. Local adult neurology services for the next decade. Report of a working party. London: RCP, 2011.

2 Weatherall MW. Acute neurology in the twenty-first century district general hospital. J R Coll Physicians Edin 2006;36:196-200.

3 Association of British Neurologists. ABN Acute Neurology services survey 2014. London: ABN, 2014.

4 Royal College of Physicians. Consultant physicians working with patients. Revised 5th edition (online update). London: RCP, 2013.

5 Federation of the Royal Colleges of Physicians of the UK. Census of consultant physicians and medical registrars in the UK, 2012: data and commentary. London: RCP, 2014.

6 Warlow C, Humphrey P, Venables G. UK neurologists and the care of adults with acute neurological problems. Clin Med 2002;2:436-9.

7 Ali E, Chaila E, Hutchinson M, Tubridy N. The 'hidden work' of a hospital neurologist: 1000 consults later. Eur J Neurol 2010;17:e28-32.

8 Costello DJ, Renganathan R, O'Hare A et al. Audit of an inpatient neurology consultation service in a tertiary referral centre: value of the consulting neurologist. Ir Med J 2005;98:134-7.

9 Moeller JJ, Kurniawan J, Gubitz GJ et al. Diagnostic accuracy of neurological problems in the emergency department. Can J Neurol Sci 2008;35:335-41.

10 Robertson NP, Shaunak S, Compston DAS. Urgent neurology out-patient referrals from primary health care physicians. QJM 1998;91:309-13.

11 Peukert T, McDonnell G, Craig J, Shortt P. Can a rapid access neurology clinic reduce A\&E admissions? Emerg Med J 2014;31:779-80.
12 Chapman FA, Pope AE, Sorensen D et al. Acute neurological problems: frequency, consultation patterns and the uses of a rapid access neurology clinic. J R Coll Physicians Edinb 2009;39:296-300.

13 Purdy S, Griffin T. Reducing hospital admissions. BMJ 2008;336:4-5.

14 Fiddes B, Adam R, Donaghy A et al. The changing face of urgent neurology outpatient referrals. J Neurol Neurosurg Psychiatry 2013;84:e2.

15 Research and Performance Team Cambridgeshire County Council. Cambridge population and dwelling stock estimates: mid 2013. Cambridge: Cambridgeshire County Council, 2014.

16 Health and Social Care Information Centre. Hospital outpatient activity 2012-2013. Leeds: HCSIS, 2013.

17 Rasmussen BK, Jensen R, Schroll M, Olesen J. Epidemiology of headache in a general population- a prevalence study. J Clin Epidemiol 1991;44:1147-57.

18 National Health Service Institute for Innovation and Improvement. Directory of ambulatory emergency care for adults. London: NHS, 2007.

19 Ridsdale L, Doherty J, McCrone P, Seed P. A new GP with special interest headache service: observational study. Br J Gen Pract 2008; $58: 478-83$.

Address for correspondence: Dr SRL Stacpoole, Addenbrooke's Hospital, Cambridge University Hospitals NHS Foundation Trust, Hills Road, Cambridge CB2 0QQ, UK.

Email: srls2@cam.ac.uk
Assessing trainees in the workplace An e-learning module for secondary care doctors

Three hours of CPD-approved interactive learning covering:

$>$ feedback

$>$ supervised learning events (SLEs)

$>$ workplace-based assessments (WPBAs)

$>$ the role of the Annual Review of Competence Progression (ARCP).

For more information please visit: www.rcplondon.ac.uk/elearning

\section{Royal College} of Physicians

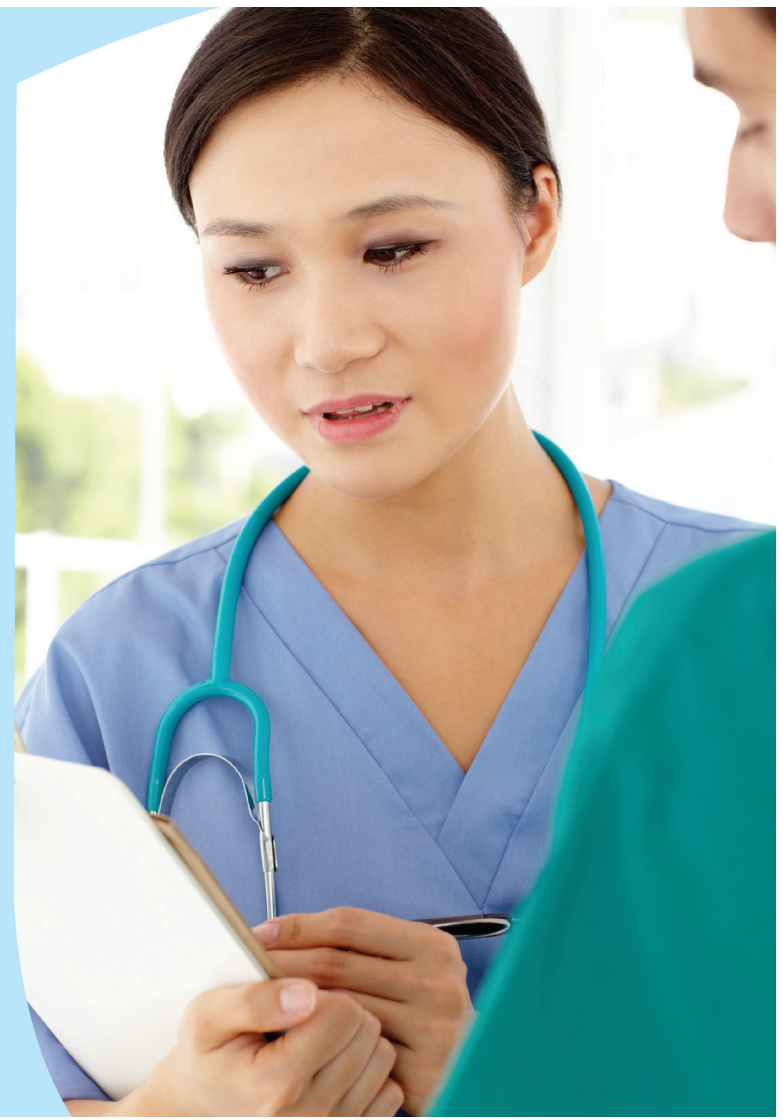

